\title{
Uncertain random assignment problem
}

DOI:

10.1016/j.apm.2017.11.026

\section{Document Version}

Accepted author manuscript

Link to publication record in Manchester Research Explorer

\section{Citation for published version (APA):}

Ding, S., \& Zeng, X. J. (2018). Uncertain random assignment problem. Applied Mathematical Modelling, 56, 96104. https://doi.org/10.1016/j.apm.2017.11.026

\section{Published in:}

Applied Mathematical Modelling

\section{Citing this paper}

Please note that where the full-text provided on Manchester Research Explorer is the Author Accepted Manuscript or Proof version this may differ from the final Published version. If citing, it is advised that you check and use the publisher's definitive version.

\section{General rights}

Copyright and moral rights for the publications made accessible in the Research Explorer are retained by the authors and/or other copyright owners and it is a condition of accessing publications that users recognise and abide by the legal requirements associated with these rights.

\section{Takedown policy}

If you believe that this document breaches copyright please refer to the University of Manchester's Takedown Procedures [http://man.ac.uk/04Y6Bo] or contact uml.scholarlycommunications@manchester.ac.uk providing relevant details, so we can investigate your claim.

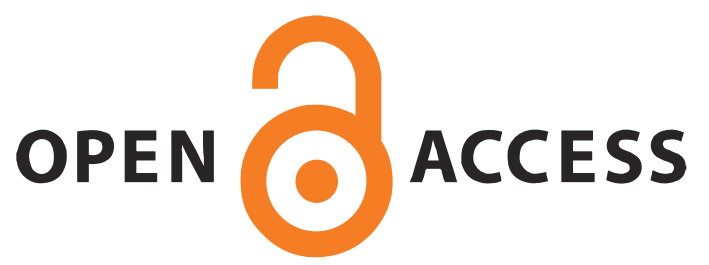




\title{
Uncertain Random Assignment Problem
}

\author{
Sibo Ding ${ }^{a, *}$ Xiao-Jun Zeng ${ }^{b}$ \\ ${ }^{a}$ School of Management, Henan University of Technology, Zhengzhou 450001, China \\ ${ }^{b}$ School of Computer Science, University of Manchester, Manchester M13 9PL, UK
}

\begin{abstract}
This paper proposes an uncertain random assignment problem in which random variables coexist with uncertain variables. Critical values of uncertain random variables are used to rank uncertain random variables. An uncertain random simulation algorithm is developed in order to obtain chance distributions and critical values of uncertain random variables. An $\alpha$-optimistic model is presented. A combined optimization approach is designed to solve the $\alpha$-optimistic model. This approach incorporates uncertain random simulation into branch and bound algorithm. Finally, an example application of the approach is presented.
\end{abstract}

Keywords:

Uncertainty Modelling, Uncertain random simulation, Uncertain variable, Uncertain random variable, Assignment problem

\section{Introduction}

The Classic Assignment Problem is one of the fundamental problems in the area of combinatorial optimization. Easterfield [1] first studied the algorithm for the Classic Assignment Problem. Kuhn [2] proposed the famous Hungarian method in order to provide the solution of classic assignment problem. Kurtzberg [3] developed approximation methods for large scale assignment problems. Other scholars have discussed the semi-assignment problem [4], the minimum deviation assignment problem [5], the fractional assignment problem [6], the k-cardinality assignment problem [7], the multidimensional assignment problem ([8-10]), the generalized assignment problem [11] and the priority based assignment problem [12].

All of the above studies assume that coefficients are constants. But, in many applications, coefficients are not constants but random variables. 
Thus, researchers study random assignment problems ([13], [14]). Mézard and Parisi [15] use the replica method to study the expected optimal value. Lazarus [16] develops the lower bound on the value of a random assignment problem. Buck et al. [17] conjecture an explicit formula for the expected value of the random k-assignment. Parviainen [18] discusses an assignment problem with discrete random costs. Li et al. [19] present a new genetic algorithm selection scheme to solve the random assignment problem. Sethuraman and Ye [20] consider the random assignment problem on a uniform preference domain. Pour et al. [21] study a new stochastic personnel assignment problem.

However, when a worker is assigned a new job, we often do not have statistical data available in order to assess his or her performance. In such a case, it is impossible to obtain the probability distributions of coefficients. However, we can invite experts to estimate coefficients based on their knowledge and experience. Such an estimation has an element of human uncertainty, as it is usually to provide an estimation. In order to deal with this human uncertainty, Liu [22] proposes an uncertain variable and establishes uncertainty theory. Thereafter, some researchers apply the uncertainty theory in order to study various uncertain problems. Yang and Ralescu [23] propose a new Adams method for solving uncertain differential equations. Hosseini and Wadbro [24] have conducted an analysis of reliability and stability in uncertain networks. Some scholars have carried out research on uncertain programming problems. For example, Dalman [25] considers an uncertain multi-objective multi-item solid transportation problem. Veresnikov et al. [26] designs an uncertain technical systems. In particular, Zhang and Peng [27] study an uncertain assignment problem in which coefficients are uncertain variables.

However, randomness and human uncertainty may coexist in complex systems. For example, when we sell new products and existing products to the existing markets, we can obtain the probability distribution of the demand for existing products from historical data. But we can not obtain the probability distribution of the demand of new products due to a lack of data. In such situations, we can invite experienced experts to estimate the demand for new products. Therefore, randomness and human uncertainty simultaneously appear in such a problem. Liu [28] proposes the use of chance theory to deal with uncertain random problems. Qin [29] then solves a portfolio selection problem with random and uncertain returns. Gao and Yao [30] discuss the uncertain random stationary increment process and the uncertain 
random renewal process.

Scholars first study deterministic models [1-12], then study models with random variables [13-21]. They then study models with uncertain variables [27]. It is therefore natural to think about how to solve the assignment problem where uncertain variables coexist with random variables. We present an uncertain random assignment model under the assumption that coefficients are independent uncertain variables and independent random variables. Since constant, random variable and uncertain variable are special cases of uncertain random variables, previous models for the assignment problem are special cases of our model. The different types of model are listed in Table 1:

Table 1. Assumption and mathematical model for assignment problem.

Assumption(coefficient) Mathematical model

\begin{tabular}{llll}
\hline deterministic model [1-12] & constant & integer programming \\
\hline random model [13-21] & $\begin{array}{l}\text { independent random } \\
\text { variable }\end{array}$ & $\begin{array}{l}\text { stochastic } \\
\text { ming }\end{array}$ & program- \\
\hline uncertain model [27] & $\begin{array}{l}\text { independent uncertain } \\
\text { variable }\end{array}$ & $\begin{array}{l}\text { uncertain } \\
\text { ming }\end{array}$ & program- \\
\hline our model & $\begin{array}{l}\text { independent uncertain } \\
\text { variable and indepen- } \\
\text { dent random variable }\end{array}$ & $\begin{array}{l}\text { uncertain random pro- } \\
\text { gramming }\end{array}$ & \\
& & & \\
\hline
\end{tabular}

Since uncertain random variables may not be differentiable and continuous, usual optimization methods cannot be used to solve uncertain random models. Researchers use simulation in order to obtain numerical solutions. Ke et al. [31] design an uncertain random simulation for solving uncertain random project scheduling problems. However, their uncertain random simulation is likely to yield different results every time. Sheng and Gao [32] propose a special algorithm for uncertain random network optimization.

In this paper, we present a new simulation algorithm. Our algorithm generates sample points uniformly, while the algorithm in [31] generates sample points stochastically. In fact, it is difficult to generate sample points 
"stochastically" for complex uncertain random variables. The algorithm in [31] is unsteady. Algorithms in [31, 32] are specially designed for project scheduling problems and network optimization problems. They can be used to simulate the chance distributions of increasing functions. However, our algorithm is general and without any restriction on functions. Our algorithm can be used to simulate chance distributions of increasing functions and decreasing functions.

The remainder of this paper is organized as follows: In Section 2, some basic concepts and theorems of uncertainty theory and chance theory are introduced. In section 3, we propose ranking criteria for the evaluation of uncertain random variables, and design an uncertain random simulation algorithm in order to obtain the chance distribution and critical values. In Section 4, we propose an uncertain random assignment problem and present the $\alpha$-optimistic model. In order to solve the corresponding optimization problem, a branch and bound algorithm integrated with uncertain random simulation is developed. Several conclusions are drawn in Section 5.

\section{Preliminary}

In this section, we introduce some concepts and theorems on uncertain theory and chance theory.

\subsection{Uncertainty Theory}

Let $\Gamma$ be a nonempty set and let $\mathcal{L}$ be a $\sigma$-algebra over $\Gamma$. A set function $\mathcal{N}: \mathcal{L} \rightarrow[0,1]$ is called an uncertain measure if it satisfies the following four normality axiom, duality axiom, subadditivity axiom and product $\operatorname{axiom}([22],[33])$.

Definition 1. (Liu [33]) The uncertain variables $\xi_{1}, \xi_{2}, \cdots, \xi_{n}$ are said to be independent if

$$
\mathcal{M}\left\{\bigcap_{i=1}^{n}\left\{\xi_{i} \in B_{i}\right\}\right\}=\bigwedge_{i=1}^{n} \mathcal{N}\left\{\xi_{i} \in B_{i}\right\}
$$

for any Borel sets $B_{1}, B_{2}, \cdots, B_{n}$.

An uncertainty distribution $\Phi(x)$ is said to be regular if it is a continuous and strictly increasing function with respect to $x$ at which $0<\Phi(x)<1$, and

$$
\lim _{x \rightarrow-\infty} \Phi(x)=0, \lim _{x \rightarrow+\infty} \Phi(x)=1 .
$$


Definition 2. (Liu [34]) Let $\xi$ be an uncertain variable with regular uncertainty distribution $\Phi(x)$. Then the inverse function $\Phi^{-1}(\alpha)$ is called the inverse uncertainty distribution of $\xi$.

The inverse uncertainty distribution of zigzag uncertain variable $\mathcal{Z}(a, b, c)$ is

$$
\Phi^{-1}(\alpha)= \begin{cases}(1-2 \alpha) a+2 \alpha b, & \text { if } \quad \alpha<0.5 \\ (2-2 \alpha) b+(2 \alpha-1) c, & \text { if } \quad \alpha \geq 0.5\end{cases}
$$

Theorem 1. (Liu [34]) Let $\xi_{1}, \xi_{2}, \cdots, \xi_{n}$ be independent uncertain variables with uncertainty distributions $\Phi_{1}, \Phi_{2}, \cdots, \Phi_{n}$, respectively. If $f\left(x_{1}, x_{2}, \cdots, x_{n}\right)$ is strictly increasing with respect to $x_{1}, x_{2}, \cdots, x_{m}$ and strictly decreasing with respect to $x_{m+1}, x_{m+2}, \cdots, x_{n}$, then $\xi=f\left(\xi_{1}, \xi_{2}, \cdots, \xi_{n}\right)$ is an uncertain variable with an inverse uncertainty distribution

$$
\Phi^{-1}(\alpha)=f\left(\Phi_{1}^{-1}(\alpha), \cdots, \Phi_{m}^{-1}(\alpha), \Phi_{m+1}^{-1}(1-\alpha), \cdots, \Phi_{n}^{-1}(1-\alpha)\right) .
$$

Theorem 2. (Liu [34]) Let $\xi_{1}, \xi_{2}, \cdots, \xi_{n}$ be independent uncertain variables with uncertainty distributions $\Phi_{1}, \Phi_{2}, \cdots, \Phi_{n}$, respectively. If $f$ is a continuous and strictly increasing function, then

$$
\xi=f\left(\xi_{1}, \xi_{2}, \cdots, \xi_{n}\right)
$$

has an uncertainty distribution

$$
\Psi(x)=\sup _{f\left(x_{1}, x_{2}, \cdots, x_{n}\right)=x} \min _{1 \leq i \leq n} \Phi_{i}\left(x_{i}\right) .
$$

\subsection{Chance Theory}

Let $(\Gamma, \mathcal{L}, \mathcal{M})$ be an uncertainty space and let $(\Omega, \mathcal{A}, \operatorname{Pr})$ be a probability space. Then the product $(\Gamma, \mathcal{L}, \mathcal{M}) \times(\Omega, \mathcal{A}, \operatorname{Pr})$ is called a chance space.

A chance distribution of an uncertain random variable $\xi$ is defined by $\Phi(x)=\operatorname{Ch}\{\xi \leq x\}$ for any $x \in \Re$.

Theorem 3. (Liu [35]) Let $\eta_{1}, \eta_{2}, \cdots, \eta_{m}$ be independent random variables with probability distributions $\Psi_{1}, \Psi_{2}, \cdots, \Psi_{m}$, respectively, and let $\tau_{1}, \tau_{2}, \cdots, \tau_{n}$ be independent uncertain variables. Assume $f$ is a measurable function. Then the uncertain random variable

$$
\xi=f\left(\eta_{1}, \eta_{2}, \cdots, \eta_{m}, \tau_{1}, \tau_{2}, \cdots, \tau_{n}\right)
$$


has a chance distribution

$$
\Phi(x)=\int_{\Re^{m}} F\left(x ; y_{1}, y_{2}, \cdots, y_{m}\right) \mathrm{d} \Psi_{1}\left(y_{1}\right) \mathrm{d} \Psi_{2}\left(y_{2}\right) \cdots \mathrm{d} \Psi_{m}\left(y_{m}\right) .
$$

where $F\left(x ; y_{1}, y_{2}, \cdots, y_{m}\right)$ is the uncertainty distribution of the variable $f\left(y_{1}, y_{2}, \cdots, y_{m}, \tau_{1}, \tau_{2}, \cdots, \tau_{n}\right)$.

\section{Ranking Criterion}

The ranking criterion for comparing uncertain random variables is a significant problem. There does not exist a natural order in an uncertain random circumstance. In order to measure it, we use critical values $(\alpha$-pessimistic value and $\alpha$-optimistic value) as ranking measures.

Definition 3. Let $\xi$ be an uncertain random variable on chance space $(\Gamma, \mathcal{L}, \mathcal{M}) \times$ $(\Omega, \mathcal{A}, \operatorname{Pr})$ and $\alpha \in(0,1]$. Then,

$$
\xi_{\text {inf }}(\alpha)=\inf \{x \mid \operatorname{Ch}\{\xi \leq x\} \geq \alpha\}
$$

and

$$
\xi_{\text {sup }}(\alpha)=\sup \{x \mid \operatorname{Ch}\{\xi \geq x\} \geq \alpha\}
$$

are called the $\alpha$-pessimistic value and the $\alpha$-optimistic value of $\xi$, respectively.

Remark 1: The $\alpha$-pessimistic value and the $\alpha$-optimistic value of random variables and uncertain variables are special cases of the $\alpha$-pessimistic value and the $\alpha$-optimistic value of uncertain random variables.

We provide the following ranking criteria.

(1) Pessimistic Value Criterion: Let $\xi$ and $\eta$ be two uncertain random variables. We say $\xi>\eta$ if and only if $\xi_{\text {inf }}(\alpha)>\eta_{\text {inf }}(\alpha)$ for some predetermined confidence level $\alpha \in(0,1]$.

(2) Optimistic Value Criterion: Let $\xi$ and $\eta$ be two uncertain random variables. We say $\xi>\eta$ if and only if $\xi_{\text {sup }}(\alpha)>\eta_{\text {sup }}(\alpha)$ for some predetermined confidence level $\alpha \in(0,1]$.

According to Definition 3, Theorems 1 and 3, we design an uncertain random simulation to obtain the chance distribution, the $\alpha$-pessimistic value and the $\alpha$-optimistic value. 


Algorithm 1
Step 1. Discretize the range of the random variable $\eta_{i}$ into $N_{i}$ equally
spaced points.

Step 2. Discretize $\alpha$ into $K$ equally spaced points.

Step 3. Calculate $\Upsilon_{1}^{-1}(\alpha), \cdots, \Upsilon_{k}^{-1}(\alpha), \Upsilon_{k+1}^{-1}(1-\alpha), \cdots, \Upsilon_{n}^{-1}(1-\alpha)$.

Step 4. Calculate $f\left(y_{1}, y_{2}, \cdots, y_{m}, \Upsilon_{1}^{-1}(\alpha), \cdots, \Upsilon_{k}^{-1}(\alpha), \Upsilon_{k+1}^{-1}(1-\alpha), \cdots\right.$, $\left.\Upsilon_{n}^{-1}(1-\alpha)\right)$. And let it equal $F^{-1}\left(\alpha ; y_{1}, y_{2}, \cdots, y_{m}\right)$.

Step 5. Let $x_{k}=F^{-1}\left(\alpha ; y_{1}, y_{2}, \cdots, y_{m}\right), 1 \leq k \leq K$.

Step 6. Use linear interpolation to compute

$$
F\left(x ; y_{1}, y_{2}, \cdots, y_{m}\right)=\left\{\begin{array}{lll}
0 & \text { if } \quad x \leq x_{1} \\
\alpha_{i}+\left(\alpha_{i+1}-\alpha_{i}\right) \frac{x-x_{i}}{x_{i+1}-x_{i}} & \text { if } \quad x_{i} \leq x \leq x_{i+1}, 1 \leq i \leq K \\
1 & \text { if } \quad x \geq x_{K} .
\end{array}\right.
$$

Step 7. Obtain the chance distribution.

Step 8. Obtain the $\alpha$-pessimistic value and the $\alpha$-optimistic value.

Since the sample points are selected uniformly from the range of variables, Algorithm 1 is stable. Our simulation algorithm does not need machine learning. So long as the sample size is large enough, the algorithm can obtain satisfactory solutions on different uncertain random variables. Time and space complexities depend on the number of variables and the sample size $\left(\sum_{i=1}^{m} N_{i}+n \cdot K\right)$. It may require much computation time and large storage space in large scale problems. However, our algorithm is very flexible. There is virtually no limit to the type of analyses that can be done. Empirical distributions also can be used.

We give the following example to illustrate Algorithm 1.

Example 1. Assume that $\eta_{1} \sim \mathcal{N}(6,4)$ and $\eta_{2} \sim U(1,2)$ are independent random variables, and $\tau_{1} \sim \mathcal{Z}(3,4,5)$ and $\tau_{2} \sim \mathcal{L}(2,3)$ are independent uncertain variables. $\xi=\eta_{1}+\tau_{1}-\eta_{2}-\tau_{2}$ is an uncertain random variable. Now we compute the chance distribution, the $\alpha$-pessimistic value and $\alpha$-optimistic value by using Algorithm 1.

The probability distributions of $\eta_{1}$ and $\eta_{2}$ are

$$
\Psi_{1}\left(y_{1}\right)=\frac{1}{\sqrt{2 \pi} \cdot 2} e^{-\frac{\left(y_{1}-6\right)^{2}}{2 \cdot 2^{2}}}
$$


and

$$
\Psi_{2}\left(y_{2}\right)=\left\{\begin{array}{lll}
0 & \text { if } & y_{2} \leq 1 \\
y_{2}-1 & \text { if } & 1 \leq y_{2} \leq 2 \\
1 & \text { if } & y_{2} \geq 2
\end{array}\right.
$$

$\Upsilon^{-1}(\alpha)$ and $\Upsilon^{-1}(1-\alpha)$ of uncertain variable $\xi$ with linear uncertain distribution $\mathcal{L}(a, b)$ are $(1-\alpha) a+\alpha b$ and $\alpha a+(1-\alpha) b$.

$$
\begin{aligned}
F^{-1}\left(\alpha ; y_{1}, y_{2}\right) & =f\left(y_{1}, y_{2}, \Upsilon_{1}^{-1}(\alpha), \Upsilon_{2}^{-1}(1-\alpha)\right) \\
& =\left\{\begin{array}{lll}
y_{1}+[(1-2 \alpha) 3+2 \alpha 4]-y_{2}-[\alpha 2+(1-\alpha) 3], & \text { if } & \alpha<0.5 \\
y_{1}+[(2-2 \alpha) 4+(2 \alpha-1) 5]-y_{2}-[\alpha 2+(1-\alpha) 3], & \text { if } & \alpha \geq 0.5 .
\end{array}\right.
\end{aligned}
$$

For simplicity's sake, we set $N_{1}=10, N_{2}=10, K=10$. In addition, according to the three sigma rule, the range of the random variable $\eta_{1}$ is $(0$, 12). Of course, the range can be set as required.

The value of $f\left(y_{1}, y_{2}, \Upsilon_{1}^{-1}(\alpha), \Upsilon_{2}^{-1}(1-\alpha)\right)$ is listed in the Table 2.

Table 2. Value of $f\left(y_{1}, y_{2}, \Upsilon_{1}^{-1}(\alpha), \Upsilon_{2}^{-1}(1-\alpha)\right)$

\begin{tabular}{cccccll}
\hline$i$ & $j$ & $k$ & $y_{1}=1.2 \cdot i$ & $y_{2}=1+0.1 \cdot j$ & $\alpha=0.1 \cdot k$ & $f$ \\
\hline 1 & 1 & 1 & 1.2 & 1.1 & 0.1 & 0.4 \\
1 & 1 & 2 & 1.2 & 1.1 & 0.2 & 0.7 \\
$\ldots$ & $\ldots$ & $\ldots$ & $\ldots$ & $\cdots$ & $\cdots$ & $\cdots$ \\
1 & 1 & 10 & 1.2 & 1.1 & 1 & 3.1 \\
\hline 1 & 2 & 1 & 1.2 & 1.2 & 0.1 & 0.3 \\
1 & 2 & 2 & 1.2 & 1.2 & 0.2 & 0.6 \\
$\ldots$ & $\ldots$ & $\ldots$ & $\ldots$ & $\ldots$ & $\ldots$ & $\cdots$ \\
1 & 2 & 10 & 1.2 & 1.2 & 1 & 3 \\
$\ldots$ & $\ldots$ & $\ldots$ & $\ldots$ & $\ldots$ & $\ldots$ & $\ldots$ \\
10 & 10 & 1 & 12 & 2 & 0.1 & 10.3 \\
10 & 10 & 2 & 12 & 2 & 0.2 & 10.6 \\
$\ldots$ & $\ldots$ & $\ldots$ & $\ldots$ & $\ldots$ & $\ldots$ & $\cdots$ \\
10 & 10 & 10 & 12 & 2 & 1 & 13 \\
\hline
\end{tabular}


The chance distribution of $\xi$ is formulated as

$$
\begin{aligned}
\Phi(x) & =\int_{1}^{2} \int_{0}^{12} F\left(x ; y_{1}, y_{2}\right) \mathrm{d} \Psi_{1}\left(y_{1}\right) \mathrm{d} \Psi_{2}\left(y_{2}\right) \\
& =\sum_{i=1}^{10} \sum_{j=1}^{10} F(x ; 1.2 \cdot i, 1+0.1 \cdot j) \cdot \frac{1}{\sqrt{2 \pi} \cdot 2} e^{-\frac{(1.2 \cdot i-6)^{2}}{2 \cdot 2^{2}}} \cdot 1.2 \cdot 0.1 .
\end{aligned}
$$

By Algorithm 1, we get the chance distribution of $\xi$ (Fig. 1).

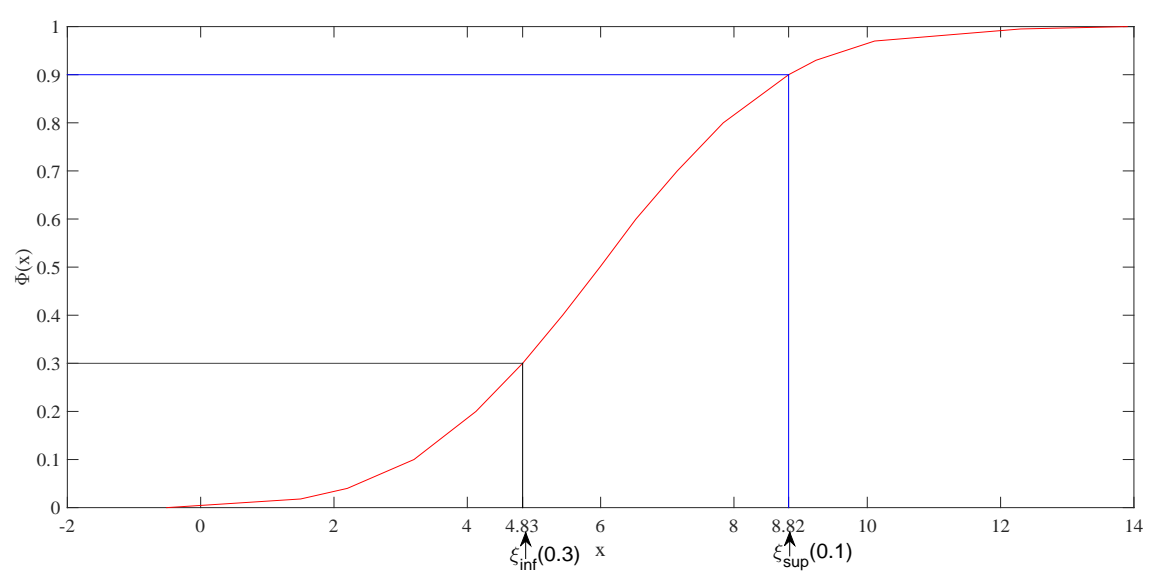

Fig. 1. The chance distribution of $\xi$.

Then, we can obtain $\Phi(4.83)=0.3$ and $\Phi(8.82)=0.9$. Thus, we can obtain the $\alpha$-pessimistic value $\xi_{\text {inf }}(0.3)=4.83$ and and the $\alpha$-optimistic value $\xi_{\text {sup }}(0.1)=8.82$.

In the next section, we will use uncertain random simulation in order to solve the uncertain random assignment problem. The $\alpha$-optimistic model is presented. Finally, we design a branch and bound algorithm to obtain the solution for the model.

\section{Uncertain random assignment model}

Given an $n \times n$ uncertain random profit matrix $\boldsymbol{\xi}=\left(\xi_{i j}\right)$ ( $n$ workers and $n$ jobs), we want to assign exactly one worker to each job and exactly one 
job to each worker so that the profit of the assignment is maximized. The uncertain random profit matrix is

$$
\boldsymbol{\xi}=\left(\begin{array}{cccc}
\xi_{12} & \xi_{12} & \cdots & \xi_{1 n} \\
\xi_{21} & \xi_{22} & \cdots & \xi_{2 n} \\
\vdots & \vdots & \ddots & \vdots \\
\xi_{n 1} & \xi_{n 2} & \cdots & \xi_{n n}
\end{array}\right)
$$

The elements of $\boldsymbol{\xi}$ are independent random variables and independent uncertain variables.

The objective function $\zeta=\sum_{i=1}^{n} \sum_{j=1}^{n} \xi_{i j} x_{i j}$ is subject to the constraints

$$
\begin{aligned}
& \sum_{j=1}^{n} x_{i j}=1, \quad i=1,2,3, \ldots, n, \\
& \sum_{i=1}^{n} x_{i j}=1, \quad j=1,2,3, \ldots, n, \\
& x_{i j}=0 \text { or } 1, \quad i, j=1,2,3, \ldots, n
\end{aligned}
$$

where $x_{i j}$ is defined as

$$
x_{i j}= \begin{cases}1 & \text { if worker } i \text { is assigned to job } j, \\ 0 & \text { otherwise }\end{cases}
$$

Since profit coefficients are uncertain variables and random variables, the assignment profit $\zeta$ is an uncertain random variable. This begs the question of how we can obtain the best assignment?

In order to solve uncertain random assignment problem, we use chance constrained programming to handle uncertainty. Since a direct comparison of uncertain random variables is meaningless, we propose a new model using optimistic value criterion (Similarly, if coefficients are costs, we can use a pessimistic value criterion). The decision-maker makes decisions in terms of $\alpha$-optimistic value $\zeta_{\text {sup }}(\alpha)$. Then, the uncertain random assignment problem can be modeled as 


$$
\begin{aligned}
& \max \zeta_{\text {sup }}(\alpha) \\
& \text { s.t. } \operatorname{Ch}\left\{\xi_{i j} \geq\left(\xi_{i j}\right)_{\sup }(\alpha)\right\} \geq \alpha \quad i=1,2,3, \ldots, n, \\
& \sum_{j=1}^{n} x_{i j}=1, \quad i=1,2,3, \ldots, n, \\
& \sum_{i=1}^{n} x_{i j}=1, \quad j=1,2,3, \ldots, n, \\
& x_{i j}=0 \text { or } 1, \quad i, j=1,2,3, \ldots, n
\end{aligned}
$$

where $\alpha$ is the confidence level that reflects the decision maker's degree of belief about an event. Constraint (7) means that each profit coefficient is greater than the $\alpha$-optimistic value with a confidence level of $\alpha$. The essential idea behind our models is to optimize some critical values with a given confidence level subject to some chance constraints.

Remark 2: Since constants, random variables and uncertain variables are special cases of uncertain random variables, it can be said that deterministic models, random models and the uncertain models are special cases of uncertain random model.

In order to obtain the solutions to the above optimization models, we first define the uncertain profit coefficient set and the random profit coefficient set as

$$
U=\left\{\tau_{i j} \mid \operatorname{cost} \text { coefficients } \xi_{i j} \text { are uncertain variables }\right\}
$$

and

$$
R=\left\{\eta_{i j} \mid \text { cost coefficients } \xi_{i j} \text { are random variables }\right\} .
$$

We can then prove the following theorem.

Theorem 4. Let $\eta_{i j}$ be independent random variables with probability distributions $\Psi_{i j}, i, j=1,2,3, \ldots, n$, and $\tau_{i j}$ be independent uncertain variables with regular uncertainty distributions $\Upsilon_{i j}, i, j=1,2,3, \ldots, n$. If $\zeta=\sum_{i=1}^{n} \sum_{j=1}^{n} \xi_{i j} x_{i j}$, then $\zeta_{\text {sup }}(\alpha)$ can be calculated by

$$
\zeta_{\text {sup }}(\alpha)=\sup \left\{x \mid \int_{\Re^{\left|\xi_{i j} \in R\right|}} F\left(x ; y_{i j}, \xi_{i j} \in R\right) \prod_{\xi_{i j} \in R} \mathrm{~d} \Psi_{i j}\left(y_{i j} x_{i j}\right) \leq 1-\alpha\right\}
$$


where

$$
F\left(x ; y_{i j}, \xi_{i j} \in R\right)=\sup _{\sum_{\xi_{i j} \in U} z_{i j} x_{i j}=x-\sum_{\xi_{i j} \in R} \sum_{i j} y_{i j}} \min _{\xi_{i j} \in R} \Upsilon_{i j}\left(z_{i j} x_{i j}\right)
$$

Proof: It follows from Theorem 2 that

$$
\begin{aligned}
& \Phi(x)=\mathrm{Ch}\left\{\sum_{i=1}^{n} \sum_{j=1}^{n} \xi_{i j} x_{i j} \leq x\right\} \\
& =\mathrm{Ch}\left\{\sum_{i=1}^{n} \sum_{j=1}^{n} \eta_{i j} x_{i j}+\sum_{i=1}^{n} \sum_{j=1}^{n} \tau_{i j} x_{i j} \leq x\right\} \\
& =\int_{0}^{1} \operatorname{Pr}\left\{\omega \in \Omega \mid \mathcal{M}\left\{\gamma \in \Gamma \mid \sum_{i=1}^{n} \sum_{j=1}^{n} \eta_{i j}(\omega) x_{i j}+\sum_{i=1}^{n} \sum_{j=1}^{n} \tau_{i j}(\gamma) x_{i j} \leq x\right\} \geq r\right\} \mathrm{d} r \\
& =\int_{0}^{1} \operatorname{Pr}\left\{\omega \in \Omega \mid \mathcal{M}\left\{\sum_{i=1}^{n} \sum_{j=1}^{n} \eta_{i j}(\omega) x_{i j}+\sum_{i=1}^{n} \sum_{j=1}^{n} \tau_{i j} x_{i j} \leq x\right\} \geq r\right\} \mathrm{d} r \\
& =\int_{\Re^{\left|\xi_{i j} \in R\right|}} \mathcal{M}\left\{\sum_{i=1}^{n} \sum_{j=1}^{n} y_{i j} x_{i j}+\sum_{i=1}^{n} \sum_{j=1}^{n} \tau_{i j} x_{i j} \leq x\right\} \prod_{\xi_{i j} \in R} \mathrm{~d} \Psi_{i j}\left(y_{i j} x_{i j}\right) \\
& =\int_{\Re^{\left|\xi_{i j} \in R\right|}} \mathcal{M}\left\{\sum_{i=1}^{n} \sum_{j=1}^{n} \tau_{i j} x_{i j} \leq x-\sum_{i=1}^{n} \sum_{j=1}^{n} y_{i j} x_{i j}\right\} \prod_{\xi_{i j} \in R} \mathrm{~d} \Psi_{i j}\left(y_{i j} x_{i j}\right) \\
& =\int_{\Re} \Upsilon\left\{x-\sum_{i=1}^{n} \sum_{j=1}^{n} y_{i j} x_{i j}\right\} \prod_{\xi_{i j} \in R} \mathrm{~d} \Psi_{i j}\left(y_{i j} x_{i j}\right)
\end{aligned}
$$

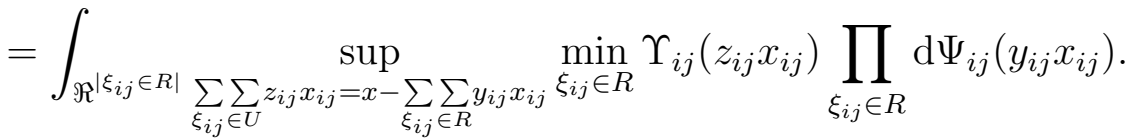

The theorem is proven.

We use optimistic value criterion to compare uncertain random variables and design Algorithm 2 to solve the uncertain random assignment problem. 


\begin{abstract}
Algorithm 2
Step 1. Set value of $\alpha$. Calculate $\alpha$-optimistic value of each element in the uncertain random profit matrix $\boldsymbol{\xi}$ and create a new matrix $\mathbf{C}$ from $\alpha$-optimistic value.

Step 2. First, select the largest elements in each row of the matrix $\mathbf{C}$. Next, select elements of $\boldsymbol{\xi}$ according to the indices of these elements. Calculate the objective value $z$ of the uncertain random model by Algorithm 1 . Then, start with the root in level 0 where the upper-bound value is equal to $z$.

Step 3. The nodes on the level $i(1 \leq i \leq n)$ of the tree correspond to selections of an element in the row of the matrix $\boldsymbol{\xi}$. Calculate the upper bound value $\zeta_{\text {sup }}(\alpha)$ for each node following best-first search strategy. Inspect each of the live nodes by comparing the upper bounds of the live nodes and terminate inferior solutions.

Step 4. Select the node that has the largest upper bound value $\zeta_{\text {sup }}(\alpha)$ as the most promising node. The job with the largest upper bound value is then assigned to the worker. Branch out from the most promising node by considering the different ways of selecting an element from the row and not in the assigned column. Inspect each of the live nodes by comparing the upper bounds of the live nodes and terminate inferior solutions.

Step 5. Repeat steps 3 to 4 till all jobs are assigned. Then, calculate the chance distribution of objective value.

Step 6. Based on the chance distribution, obtain the lower bound and the upper bound of objective value.
\end{abstract}

Because Algorithm 2 is seeking all possible solutions by using branch and bound to find the best solution, this algorithm is stable by nature. Since our algorithm integrates uncertain random simulation, calculation time and storage space are also proportional to the number of variables and sample size.

We next provide a numerical example to illustrate how to solve uncertain random assignment problems by using Algorithm 2.

\title{
Example 2.
}

We apply our algorithm to the problem of assigning three students to three tasks so that the total score of the assignment is as large as possible.

Students have formed teams for the Logistics Simulation Contest in Henan Province, China. Each team is made up of three students. First of all, they are required to take part in the preliminary contest of the University. The 
teacher grades the academic ability, organizational ability and presentation ability of all three students, based on their academic records and performances in the preliminary contest of the University. Since we have historical data of academic records, we use probability distributions of academic records to represent the students' academic ability. However, because there is no data demonstrating students' organizational ability and presentation ability, we cannot obtain the probability distributions for these two abilities. But, according to students' preliminary contest performances, we can provide uncertainty distributions of organizational ability and presentation ability based on our knowledge. Hence, it is shown that randomness and human uncertainty coexist in the assignment problem.

Students with different special abilities will perform different tasks. Students with high academic ability are mainly responsible for the writing of simulation solutions. Students with good organizational ability are mainly responsible for team leadership and communication. Those students with strong presentation abilities are mainly given responsibility for the presentation of solutions in the contest.

Next, we calculate the chance distribution of each team's score, compare the scores of different teams with the same confidence levels and select the best team to represent the University. We choose the data from the best team in order to illustrate our algorithm. In fact, this team won the championship in the subsequent provincial competition. The data are listed in the following Table 3.

Table 3. Probability distributions and empirical uncertainty distributions.

\begin{tabular}{clll}
\hline Student & Academic ability & Organizational ability & Presentation ability \\
\hline A & $\mathcal{N}(82.4,5.7)$ & $\mathcal{Z}(86,90,95)$ & $\mathcal{L}(82,90)$ \\
$\mathrm{B}$ & $\mathcal{N}(84.2,4.9)$ & $\mathcal{Z}(90,95,98)$ & $\mathcal{L}(92,99)$ \\
$\mathrm{C}$ & $\mathcal{N}(84.2,4.9)$ & $\mathcal{L}(80,88)$ & $\mathcal{L}(91,96)$ \\
\hline
\end{tabular}

We follow the steps of Algorithm 2 in order to obtain the optimal solution of the $\alpha$-optimistic model.

Step 1. We set $\alpha=0.2$, calculate $\alpha$-optimistic values of matrix $\boldsymbol{\xi}$ and 
create a new matrix $C$

$$
\boldsymbol{C}=\left(\begin{array}{ccc}
84.41 & 93 & 88.4 \\
86.05 & 96.8 & 97.6 \\
94.08 & 86.4 & 95
\end{array}\right)
$$

Step 2. We choose the maximum element in each row of matrix $\boldsymbol{C}$. They are $c_{12}, c_{23}$ and $c_{33}$. Thus, we select $\xi_{12}, \xi_{23}$ and $\xi_{33}$ from matrix $\boldsymbol{\xi}$ to calculate the objective value $z=285.6$. Level 0 is the root.

Step 3. For any legitimate selection that selects $\xi_{11}$ from the first row, the upper bound is the $\alpha$-optimistic value of $\xi_{11}+\xi_{23}+\xi_{33}$. The upper bound of node 1 is 275.4. We apply the same thinking in order to obtain the upper bounds of node $2\left(\xi_{12}+\xi_{33}+\xi_{33}\right)$ and node $3\left(\xi_{13}+\xi_{22}+\xi_{31}\right)$. They are 285.6 and 277.7 .

Since node 3 corresponds to a feasible solution and the cost of node 1 is lower than the cost of the solution represented by node 3 , node 1 is thus terminated.

Step 4. Because node 2 has the maximum upper bound value, it is the most promising node. We branch out from node 2 by considering the two different ways of selecting an element from row 2, and not in the first column.

Step 5. Of the three live leaves (nodes 3, 4 and 5), node 5 corresponds to a feasible solution with the maximum total cost of 283.1. Node 3 and node 4 are terminated. A complete state-space tree for the instance of the assignment problem is presented in Fig. 2.

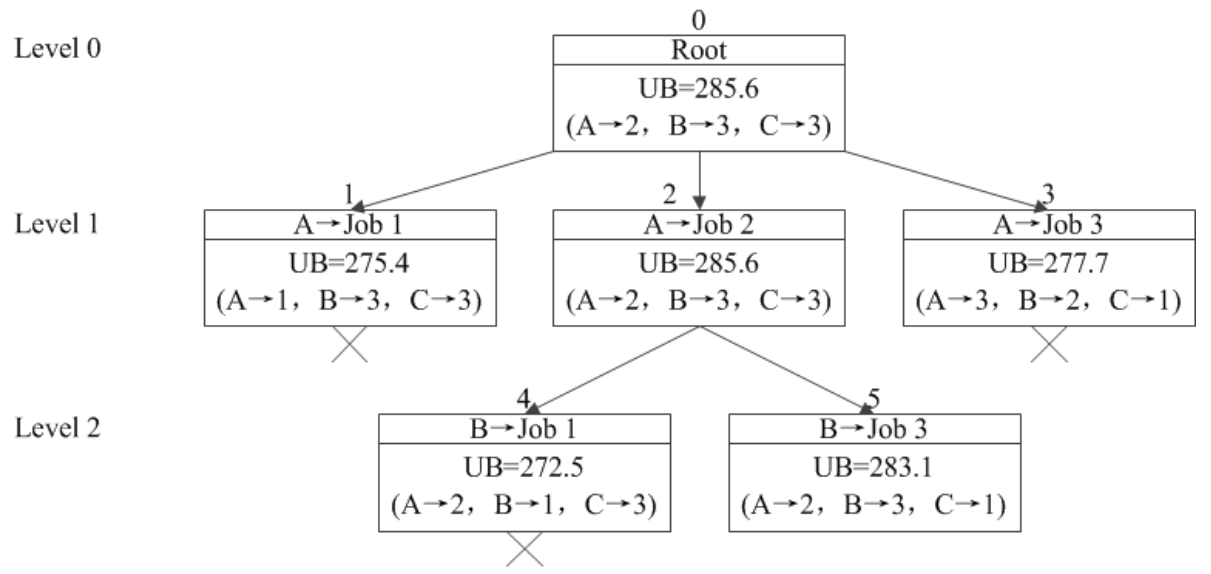

Fig. 2. State space tree. 
The optimal solution is $\{$ Student $\mathrm{A} \rightarrow$ task 2 , Student $2 \rightarrow$ task 3 , Student $3 \rightarrow$ task 1$\}$ with $\xi_{\text {sup }}(0.2)=283.1$. This means that the team has a $20 \%$ chance of obtaining a score that is greater than 283.1.

We can obtain the chance distribution of the score with different confidence level $\alpha$. Fig. 3 shows the chance distribution.

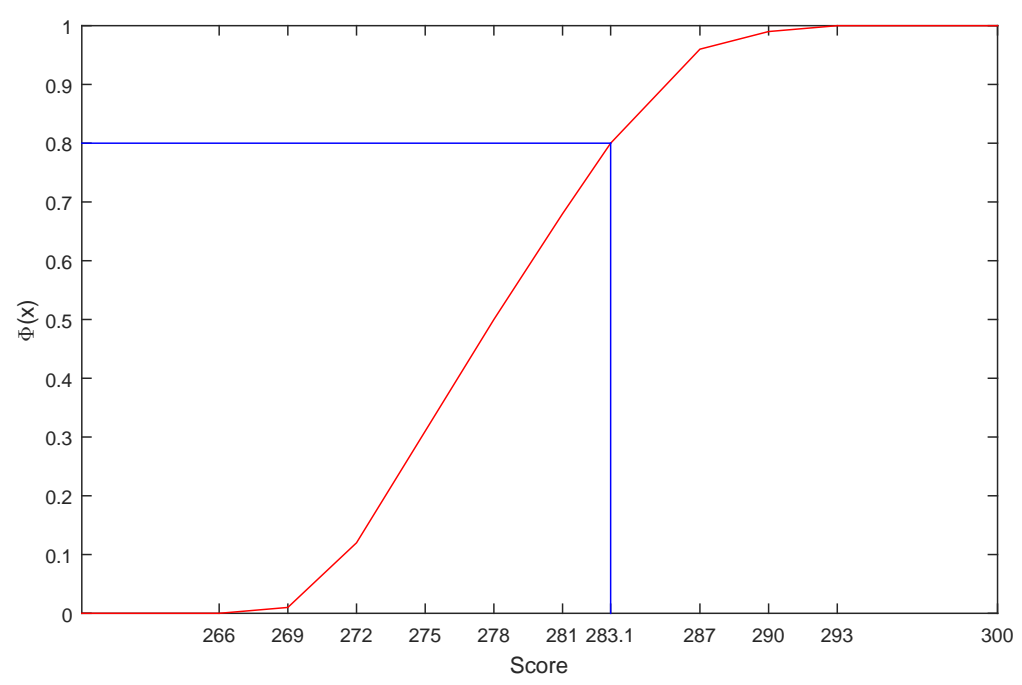

Fig. 3. Chance distribution of score

From Fig. 3, we obtain the lower bound score $266(\alpha=0)$ and the upper bound 293 score $(\alpha=1)$. They are the lowest possible score and highest possible score of the team.

\section{Conclusion}

This paper studies the uncertain random assignment problem in which some coefficients are random variables and others are uncertain variables. In order to solve the uncertain random assignment problem, we propose an optimistic value criterion and design an uncertain random simulation algorithm. Our uncertain random simulation algorithm can be used to compute chance distributions and critical values for all uncertain random variables. It can be commonly used in uncertain random optimization. 
In addition, an $\alpha$-optimistic model for uncertain random assignment problem is proposed. In order to solve this $\alpha$-optimistic model, a branch and bound algorithm combined with uncertain random simulation has been developed. It can obtain the lower bound and upper bound of the objective value. A real-world example is presented in order to illustrate the algorithm. The limitation of our algorithm lies in the fact that it needs a lot of storage space and involves a lot of calculation time. In the future, we will aim to design other superior algorithms in order to solve uncertain random problems.

\section{Acknowledgments}

This work was supported by the National Natural Science Foundation of China (Grant No. U1404701), the Scholarship Programm of China Scholarship Council (Grant No. 201509895007) and the Fundamental Research Funds for the Universities in Henan Province (Grant No. 2017RCJH11).

\section{References}

[1] T. E. Easterfield, A combinatorial algorithm, J. Lond. Math. Soc. 21 (3) (1946) 219-226.

[2] H. W. Kuhn, The Hungarian method for the assignment problem, Naval Res. Logist. Quart. 2 (1-2) (1955) 83-97.

[3] J. M. Kurtzberg, On approximation methods for the assignment problem, J. ACM. 9 (4) (1962) 419-439.

[4] R. S. Barr, F. Glover, D. Klingman, A new alternating basis algorithm for semi-assignment networks. In W. White, editor, Computers and Mathematical Programming, National Bureau of Standards Special Publications, US Government Printing Office, Washington, DC, 1978, pp. 223-232.

[5] S. K. Gupta, A. P. Punnen, Minimum deviation problems, Oper. Res. Lett. 7 (4) (1988) 201-204.

[6] M. Shigeno, Y. Saruwatari, T. Matsui, An algorithm for fractional assignment problems, Discrete Appl. Math. 56 (2-3) (1995) 333-343.

[7] M. Dell'Amico, S. Martello, The k-cardinality assignment problem, Discrete Appl. Math. 76 (1-3) (1997) 103-121. 
[8] M. Vlach, Branch and bound method for the three-index assignment problem, Economicko-Matematicky Obzor, 3 (1967) 181-191.

[9] R. E. Burkard, E. Çela, Linear assignment problems and extensions. In: Du, D.-Z., Pardalos, P.M. (Eds.), In: Handbook of Combinatorial Optimization, vol. A (Suppl.). Kluwer Academic Publishers, Dordrecht, 1999, pp. $75-149$.

[10] R. E. Burkard, Selected topics on assignment problems, Discrete Appl. Math. 123 (1-3) (2002) 257-302.

[11] S. O. Krumke, C. Thielen, The generalized assignment problem with minimum quantities, Eur. J. Oper. Res. 228 (1) (2013) 46-55.

[12] P. Kaur, A. Sharma, V. Verma, K. Dahjya, A priority based assignment problem, Appl. Math. Model. 40 (17-18) (2016) 7784-7795.

[13] W. E. Donath, Algorithm and average-value bounds for assignment problems, IBM J. Res. Dev. 13 (4) (1969) 380-386.

[14] D. W. Walkup, On the expected value of a random assignment problem, SIAM J. Comput. 8 (3) (1979) 440-442.

[15] M. Mézard, G. Parisi, Replicas and optimization. J. Phys. Lett, 46 (17) (1985) 771-778.

[16] A. J. Lazarus, Certain expected values in the random assignment problem. Oper. Res. Lett, 14 (4) (1993) 207-214.

[17] M. W. Buck, C. S. Chan, D. P. Robbins, On the expected value of the minimum assignment, Random Struct Algor, 21 (1) (2002) 33-58.

[18] Parviainen, Random assignment with integer costs, Comb. Probab. Comput. 13 (1) (2004) 103-113.

[19] F. C Li, L. D. Xu, C. X. Jin, H. Wang, Random assignment method based on genetic algorithms and its application in resource allocation, Expert Syst. Appl. 39 (15) (2012) 12213-12219.

[20] J. Sethuraman, Ch. Ye, A note on the assignment problem with uniform preferences, Oper. Res. Lett. 43 (3) (2015) 283-287. 
[21] A. G. Pour, Z. Naji-Azimi, M. Salari, Sample average approximation method for a new stochastic personnel assignment problem, Comput. Ind. Eng. 2017, doi: http://dx.doi.org/10.1016/j.cie.2017.09.006.

[22] B. Liu, Uncertainty Theory, second ed., Springer, Verlag, Berlin, 2007.

[23] X. F. Yang, D. A. Ralescu, Adams method for solving uncertain differential equations, Appl. Math. Comput. 270 (2015) 993-1003.

[24] S. A. Hosseini, E. Wadbro, Connectivity reliability in uncertain networks with stability analysis, Expert Syst. Appl. 57 (2016) 337-344.

[25] H. Dalman, Uncertain programming model for multi-item solid transportation problem, Int. J. Mach. Learn. Cyb. 2016, DOI: 10.1007/s13042016-0538-7.

[26] G. S. Veresnikov, L. A. Pankova, V. A. Pronina, Uncertain programming in preliminary design of technical systems with uncertain parameters, Procedia Comput. Sci. 103 (2017) 36-43.

[27] B. Zhang, J. Peng, Uncertain programming model for uncertain optimal assignment problem, Appl. Math. Model. 37 (9) (2013) 6458-6468.

[28] Y. H. Liu, Uncertain random variables: A mixture of uncertainty and randomness, Soft Comput. 17(4) (2013) 625-634.

[29] Z. F. Qin, Mean-variance model for portfolio optimization problem in the simultaneous presence of random and uncertain returns, Eur. J. Oper. Res. 245 (2) (2015) 480-488.

[30] J. W. Gao, K. Yao, Some concepts and theorems of uncertain random process, Int. J. Intell. Syst. 30 (1) (2015) 52-65.

[31] H. Ke, H. M. Liu, G. D. Tian, An uncertain random programming model for project scheduling problem, Int. J. Intell. Syst. 30 (1) (2015) 66-79.

[32] Y. H. Sheng, Y. Gao, Shortest path problem of uncertain random network, Comput. Ind. Eng. 99 (2016) 97-105.

[33] B. Liu, Some research problems in uncertainty theory, J. Uncertain Syst. 3 (1) (2009) 3-10. 
[34] B. Liu, Uncertainty Theory: A Branch of Mathematics for Modeling Human Uncertainty, Springer, Verlag, Berlin, 2010.

[35] Y. H. Liu, Uncertain random programming with applications, Fuzzy Optim. Decis. Ma. 12 (2) (2013) 153-169. 